\title{
LA UNIVERSIDAD EN EL DESARROLLO DEL EMPRENDIMIENTO A TRAVÉS DE UNA PLATAFORMA VIRTUAL EN EL CECADEL DE LA UNESUM - PAJAN
}

\section{\begin{tabular}{lllllll}
\hline THE UNIVERSITY IN THE DEVELOPMENT OF THE & OF
\end{tabular} ENTREPRENEURSHIP THROUGH A VIRTUAL PLATFORM IN THE CECADEL OF UNESUM - PAJAN}

\author{
Christian Ruperto Caicedo Plúa ${ }^{1}$ \\ Antonieta Del Carmen Rodríguez Gonzalez ${ }^{2}$ \\ Martha Irene Romero Castro ${ }^{3}$ \\ Eduardo Lino Rodríguez ${ }^{4}$
}

1. Magister en Gerencia Educativa e Investigación, Ingeniero en Computación y Redes, Investigador Acreditado por la Senescyt REG-INV-16-01626, Docente Titular Principal de la Universidad Estatal del Sur de Manabí, Carrera Ingeniería en Computación y Redes, Comisión Científica de la Carrera Ingeniería en Computación y Redes, Consejo Científico de la Universidad Estatal del Sur de Manabí. E-mail: christiancaicedoplua@hotmail.com

2. Magister en enseñanza del idioma Ingles, Licenciada en Ciencia de la Educación mención Ingles, Tecnóloga en Computación Administrativa, Docente Titular Principal de la Universidad Estatal del Sur de Manabí, Carrera Ingeniería en Computación y Redes, Comisión Científica de la Carrera Ingeniería en Computación y Redes.

E-mail: antojipi2006@hotmail.com

3. Magister en Informática Empresarial, Magister en Docencia Universitaria e Investigación Educativa, Ingeniero en Sistemas, Docente Titular Principal de la Universidad Estatal del Sur de Manabí, Carrera Ingeniería en Computación y Redes, Comisión Científica de la Carrera Ingeniería en Computación y Redes E-mail: mireneromero@hotmail.com

4. Ingeniero en Computación y Redes. E-mail: eduardolino@hotmail.com

\section{Citación sugerida:}

Caicedo Plúa, C.R., Rodríguez González, A.C, Romero Castro, M.I. y Rodríguez, E.L. (2016). La Universidad en el desarrollo del emprendimiento a través de una plataforma virtual en el cecadel de la UNESUM - Paján. 3C Tecnología: glosas de innovación aplicadas a la pyme, 5(4), 36-55. DOI: <http://dx.doi.org/10.17993/3ctecno.2016.v5n4e20.36-55/>. 


\section{RESUMEN}

El objeto del presente artículo de investigación consiste en establecer un modelo de enseñanza enfocada a resolver las necesidades de aprendizaje para 352 productores a través de una plataforma virtual en el CECADEL del Cantón Paján. Se realizó una investigación cuantitativa y cualitativa de tipo experimental, descriptivo y documental mediante técnicas como la encuesta y entrevista. Durante el proceso se diagnosticó la demanda de formación, investigación e innovación tecnológica y se establecieron características de las plataformas virtuales de aprendizaje. Se concluyó con el desarrollo, implementación y evaluación de las mismas para el fortalecimiento de la gestión del conocimiento acorde con las exigencias y políticas públicas que demanda el Gobierno nacional del Ecuador a través del Plan Nacional del buen vivir, objetivo 10, lo que permite mejorar la calidad del producto, superar niveles de analfabetismo digital y por ende potenciar el desarrollo de la economía local.

\section{ABSTRACT}

The objective of this research article was to establish a teaching model focused on solving the learning needs of 352 producers through a virtual platform in the CECADEL of the Paján Canton, a quantitative and qualitative research of experimental, descriptive and Documentary through techniques such as the survey and interview. During the process the demand for training, research and technological innovation was diagnosed, characteristics of virtual learning platforms were determined and concluded with the development, implementation and evaluation of the same for the strengthening of The management of knowledge in accordance with the demands and public policies demanded by the National Government of Ecuador through the National Plan of good living, objective 10, which allows to improve the quality of the product, overcome levels of digital illiteracy and thus enhance development Of the local economy.

\section{PALABRAS CLAVE}

Enseñanza virtual, matriz productiva, innovación tecnológica, gestión del conocimiento, plan nacional del buen vivir.

\section{KEY WORDS}

Virtual teaching, productive matrix, technological innovation, knowledge management, national plan of good living. 


\section{INTRODUCCIÓN}

En Latinoamérica, las plataformas virtuales van tomando mayor impulso y popularidad entre las personas. Gran parte de los países las están utilizando para fortalecer la enseñanza en todos los niveles, de hecho, las metodologías y técnicas utilizadas para el desarrollo de competencias específicas conlleva a desarrollar estrategias en base a recursos tecnológicos cooperativos y colaborativos para mejorar la comunicación en el proceso enseñanza / aprendizaje de acuerdo al área específica. Las A.V. son comúnmente utilizadas para la creación y desarrollo de módulos didácticos online y se utilizan de manera más amplia en la Web 2.0. Entre las ventajas más significativas está el ahorro en gastos de libros y material para escribir, también se reducen los tiempos de transferencia de información en los usuarios que utilizan las plataformas virtuales didácticas, se utilizan diversos tipos de dispositivos digitales para acceder a las plataformas y se utilizan diferentes recursos didácticos, tales como: videos, audios, libros electrónicos, pruebas digitales, que permitirán realizar un seguimiento y evaluación a los educandos que utilizan este tipo de medios de aprendizaje.

Desde esta perspectiva podemos relacionar diferentes aspectos científicos y académicos que denotan características epistemológicas de la concepción aprender - haciendo. Requena, (2008) vincula la educación a distancia con el constructivismo, ya que brinda un nuevo paradigma a través del uso de las nuevas tecnologías, pues los estudiantes no sólo tienen a su alcance el acceso a un mundo de información ilimitada de manera instantánea, sino que también se les ofrece la posibilidad de controlar la dirección de su propio aprendizaje. Desde este punto de vista, el objetivo principal es transformar el esquema mental en el aula en donde se adquiera una experiencia única para la construcción de nuevos conocimientos, de tal forma que una excelente educación ligada al aporte significativo de entidades públicas y privadas conllevará al tan ansiado desarrollo social, económico, científico y cultural de los pueblos. De aquí que IAEN (2011) asevera que la relación entre conocimiento y políticas públicas se enmarca en la producción de nuevos conocimientos para beneficio social.

En el caso de Ecuador, es pertinente ubicar la cuestión en el contexto actual, donde las políticas públicas deben enmarcarse en un Plan Nacional de Desarrollo local como modo de gestión de los recursos públicos para alcanzar en Buen Vivir. La constitución de la República del Ecuador en sus artículos 280, 319 y 320 reconocen y garantizan las diversas formas de organización de los procesos de producción donde se estimula la gestión participativa, transparente y eficiente. La producción, en cualquiera de sus formas, se sujetará a principios y normas de calidad, sostenibilidad, productividad sistémica, valoración del trabajo y eficiencia económica y social, de igual manera el desarrollo de ciudades inteligentes.

La Universidad Estatal del Sur de Manabí en el 2015 define ser una de las IES nivel del Ecuador pioneras en instituir los Centros de Capacitación y Desarrollo Local (CECADEL), demostrando que el trabajo en cooperación con los Gobiernos Autónomos Descentralizados podrá sistematizar procesos para alcanzar mejores resultados en las gestiones operativas, técnicas y científicas para el desarrollo local. El objetivo principal de la E-Learning es facilitar procesos de enseñanza aprendizaje para 
profesionales, agricultores, artesanos, ganaderos, estudiantes y así generar una manera sostenible el Buen vivir de los ciudadanos y ciudadanas (Torres, Prieto, y López, 2012).

\section{METODOLOGÍA}

El proyecto tiene un enfoque estratégico que ha permitido beneficiar al GAD del Cantón Paján, lo cual ha consistido en diseñar la Plataforma virtual para desarrollar destrezas en diferentes aristas para beneficio de la comunidad, logrando fortalecer la participación ciudadana bajo un modelo de cogestión y co-responsabilidad enfocados a alcanzar el buen vivir, lo que permite potenciar políticas públicas mediante el uso de las TI. Se ha realizado una investigación experimental, descriptiva y documental mediante técnicas cuantitativas y cualitativas para la descripción de los datos, permitiendo capacitar a profesionales, agricultores y con el desarrollo de seminarios virtuales a ganaderos, estudiantes y la ciudadanía en general. Se ha trabajado con encuestas y entrevistas que han permitido identificar la necesidad de capacitación en áreas específicas de manera técnica para el desarrollo productivo local, y se ha establecido la demanda de formación, investigación e innovación científica enfocando las nuevas tendencias tecnológicas, lo que ha permitido que los Centros de Capacitación y Desarrollo Local sean eficientes y dinámicos. Este medio virtual se convierte en un sistema muy factible y agradable acorde a los avances técnicos - científicos y metodológicos cuyo aporte significativo nace de la Universidad Estatal del Sur de Manabí, facultad Ciencias Técnicas, Carrera de Ingeniera en Computación y Redes, Comisión de Investigación, Consejo Científico de la IES, y Gad Paján. Ing. Ch.R.C.P.

El vertiginoso avance de los sistemas de información y comunicaciones en el Ecuador ha facilitado la automatización de los procesos, brindando confiabilidad, velocidad y precisión en el manejo de cada proceso automatizado. Desde este enfoque se puede decir que el uso eficiente y oportuno de la tecnología en procesos de capacitación para articular la gestión del conocimiento al sector productivo, define una gran ventaja para un desarrollo integral y participativo por parte de los productores identificados en el desarrollo de este proyecto.

\section{RESULTADOS Y DISCUSIÓN}

A través del método de la encuesta dirigida a productores y empleados del Gad Paján se pudo identificar la necesidad de solucionar el hecho científico ya que resulta necesaria la búsqueda de estrategias para fortalecer el desarrollo local de acuerdo al Plan Nacional del Buen Vivir, el PDyOT y la Constitución de la República del Ecuador.

1. ¿Considera usted que la dependencia económica que presenta el sector productivo afecta en los conocimientos y en las innovaciones tecnológicas?

Tabla 1. Dependencia económica que presenta el sector productivo.

\begin{tabular}{|c|r|c|}
\hline Alternativa & Frecuencia & \multicolumn{1}{|c|}{$\%$} \\
\hline Sí & 198 & 56 \\
\hline No & 154 & 44 \\
\hline Total & 352 & 100 \\
\hline
\end{tabular}

Fuente: Encuesta a productores y empleados del Gad Paján. 


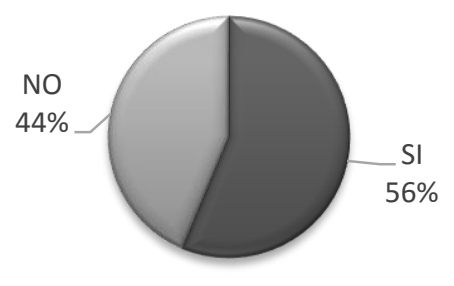

$$
\square \mathrm{SI} \square \mathrm{NO}
$$

Gráfico 1. Dependencia económica que presenta el sector productivo.

Los resultados indican que el $56 \%$ de los productores y empleados del Gad Paján tienen una dependencia económica del sector productivo e influye en los conocimientos y en las innovaciones tecnológicas, mientras que el $44 \%$ sostiene que esta dependencia no se ve negativamente reflejada en los conocimientos.

Obteniendo estos resultados se llega a la conclusión de que los productores y empleados del Gad Paján están conscientes de que la dependencia económica sí afecta en los conocimientos e innovaciones tecnológicas debido a que al depender económicamente de su producción y al no ser ésta de excelente calidad carecerían del dinero necesario para fortalecer sus conocimientos utilizando nuevas tecnologías de alcance.

2. ¿Considera usted de gran importancia el rol protagónico que tiene la universidad al brindar capacitaciones en áreas estratégicas para el desarrollo local en el cantón Paján?

Tabla 2. La universidad como rol protagónico brindando capacitación.

\begin{tabular}{|c|r|c|}
\hline Alternativa & Frecuencia & $\%$ \\
\hline Sí & 238 & 68 \\
\hline No & 114 & 32 \\
\hline Total & 352 & 100 \\
\hline
\end{tabular}

Fuente: Encuesta a productores y empleados del Gad Paján.

Elaborado por: Investigadores.

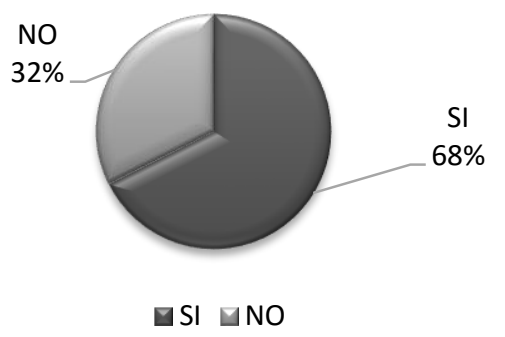

Gráfico 2. La universidad como rol protagónico brindando capacitación. 
Los resultados indican que el $68 \%$ de los productores y empleados del Gad Paján consideran que la Universidad cumple un rol protagónico al brindar capacitaciones tecnológicas, mientras que el 32\% sostiene que no es relativamente importante.

Este resultado nos muestra que el rol protagónico que cumple la Universidad al brindar capacitaciones tecnológicas es corroborado con la respuesta de la mayoría de los encuestados que piensan que es de mucha trascendencia la preparación en áreas estratégicas debido a que teniendo buena formación ésta se convertirá en una puerta abierta para el desarrollo del cantón Paján.

\section{3. ¿Cree usted que la falta de capacitación al sector productivo es un factor primordial en la baja} calidad de sus productos?

Tabla 3. Factor primordial en la baja calidad de sus productos.

\begin{tabular}{|c|c|c|}
\hline Alternativa & Frecuencia & $\%$ \\
\hline Sí & 300 & 85 \\
\hline No & 52 & 15 \\
\hline Total & 352 & 100 \\
\hline
\end{tabular}

Fuente: Encuesta a productores y empleados del Gad Paján.

Elaborado por: Investigadores.

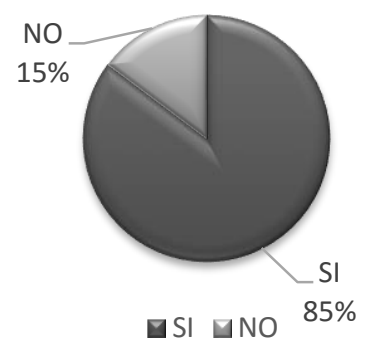

Gráfico 3. Factor primordial en la baja calidad de sus productos.

Los resultados indican que el $85 \%$ de los productores y empleados del Gad definen que existe una falta de formación que afecta la calidad de sus productos, mientras que el $15 \%$ indica que no es importante la formación para obtener buenos servicios.

Al interpretar los resultados obtenidos se llega a la conclusión de que la mayoría de los productores y empleados del Gad Paján opinan que es necesario cursos de capacitación para el sector productivo con la finalidad de obtener una dependencia económica y que de esta manera mejoren sus productos y puedan ser competitivos a nivel local, nacional e internacional.

4. ¿Cree usted que el sector productivo debería tener una mejor formación y capacitación para tener una dependencia económica y así fortalecer la matriz productiva?

Tabla 4. Sector productivo con una mejor formación de capacitación.

\begin{tabular}{|c|c|l|}
\hline Alternativa & Frecuencia & $\%$ \\
\hline Sí & 272 & 77 \\
\hline No & 80 & 23 \\
\hline Total & 352 & 100 \\
\hline
\end{tabular}

Fuente: Encuesta a productores y empleados del Gad Paján Elaborado por: Investigadores. 


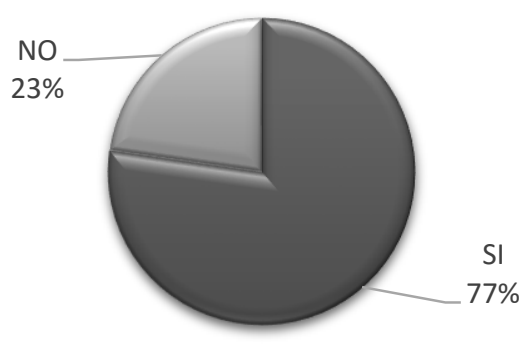

$\square \mathrm{SI} \square \mathrm{NO}$

Gráfico 4. Sector productivo con una mejor formación de capacitación.

Los resultados indican que el $77 \%$ deberían tener una mejor formación para obtener dependencia económica, mientras que el $23 \%$ sostiene que no es necesaria la formación para este fin.

Estos resultados muestran que los productores y empleados del Gad Paján tienen que obtener una dependencia económica. Es imprescindible una mejor formación para así lograr fortalecer la matriz productiva que conllevará al desarrollo económico del cantón Paján.

5. ¿Considera usted necesaria la continua capacitación del sector productivo para obtener un mejor desarrollo local en el Cantón Paján?

Tabla 5. Mejora el desarrollo local del Cantón Paján.

\begin{tabular}{|c|c|c|}
\hline Alternativa & Frecuencia & $\%$ \\
\hline Sí & 300 & 85 \\
\hline No & 52 & 15 \\
\hline Total & 352 & 100 \\
\hline
\end{tabular}

Fuente: Encuesta a productores y empleados del Gad Paján.

Elaborado por: Investigadores.

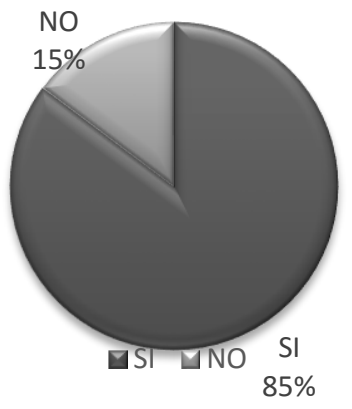

Gráfico 5. Mejora el desarrollo local del Cantón Paján.

Los resultados indican que el $88 \%$ de los encuestados necesitan cursos de capacitación, mientras que el $15 \%$ sostiene que no que es necesario una capacitación en el sector productivo.

En conclusión, se puede indicar que en la actualidad el sector productivo necesita de una continua capacitación para obtener una mejor producción y así ampliar sus conocimientos gracias al Centro de Capacitación y Desarrollo Local que vendrían a repercutir en el desarrollo local del cantón Paján. 
6. ¿Cree usted que la cadena productiva de paja mocora, café, maíz, y ganadería bovina debería tener un plan estratégico para el desarrollo local?

Tabla 6. Producción en plan estratégico.

\begin{tabular}{|c|c|c|}
\hline Alternativa & Frecuencia & $\%$ \\
\hline Sí & 280 & 80 \\
\hline No & 72 & 20 \\
\hline Total & 352 & 100 \\
\hline
\end{tabular}

Fuente: Encuesta a productores y empleados del Gad Paján.

Elaborado por: Investigadores.

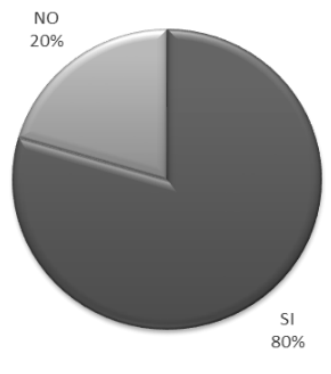

$\square \mathrm{SI} \square \mathrm{NO}$

Gráfico 6. Producción en plan estratégico.

Los resultados indican que el $80 \%$ se cree que es necesario una un plan estratégico de producción, mientras que el $20 \%$ sostiene que no es necesario.

Haciendo el análisis a los resultados obtenidos, se puede concluir que la cadena productiva de paja mocora, café, maíz y ganadería boina debe poseer un plan estratégico para el dar un mejor alcance al desarrollo local del cantón Paján para que pueda convertirse en un potencial económico a nivel provincial y nacional.

7. ¿Está usted de acuerdo con la elaboración del curso online sobre agricultura orgánica para el sector productivo mediante una plataforma virtual?

Tabla 7. Elaboración de cursos online.

\begin{tabular}{|c|c|l|}
\hline Alternativa & Frecuencia & $\%$ \\
\hline Sí & 352 & 100 \\
\hline No & 0 & 0 \\
\hline Total & 352 & 100 \\
\hline
\end{tabular}

Fuente: Encuesta a productores y empleados del Gad Paján.

Elaborado por: Investigadores. 


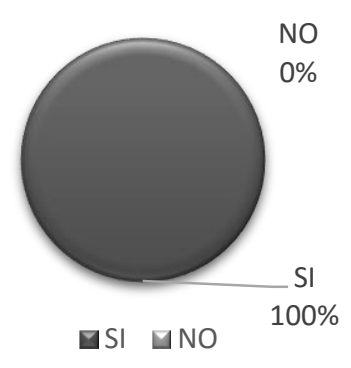

Gráfico 7. Elaboración de cursos online.

Los resultados indican que el 100\% de la productores y empleados del Gad Paján sí están de acuerdo con el curso online de agricultura orgánica.

Analizando los resultados obtenidos a la encuesta podemos afirmar que para el sector productivo es muy importante la agricultura orgánica, ya que es un sistema de manejo racional de los recursos naturales, sin la utilización de síntesis química y de esta forma se puede brindar alimentos sanos y abundantes, es así que acceder a un curso on-line sobre este tema resulta de gran importancia y mucho más factible si es a través de una plataforma virtual la cual les ahorraría tiempo y dinero.

8. ¿Considera usted que el Gobierno Autónomo Descentralizado del Cantón Paján elabore plan estratégico para potenciar la cadena productiva en general y el turismo?

Tabla 8. Cadena productiva.

\begin{tabular}{|c|c|l|}
\hline Alternativa & Frecuencia & $\%$ \\
\hline Sí & 288 & 18 \\
\hline No & 64 & 82 \\
\hline Total & 352 & 100 \\
\hline
\end{tabular}

Fuente: Encuesta a productores y empleados del Gad Paján.

Elaborado por: Investigadores.

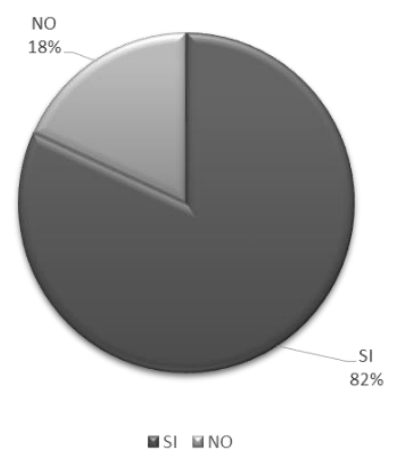

Gráfico 8. Cadena productiva.

Los resultados indican que el $82 \%$ de los productores y empleados del Gad Paján sí están de acuerdo con el plan estratégico para el fortalecimiento del turismo, mientras $18 \%$ no está de acuerdo con este plan.

En conclusión, el Gobierno Autónomo Descentralizado del Cantón Paján debe planificar un plan estratégico para potenciar la cadena productiva así como también estrategias para potenciar el 
turismo lo que permitirá elevar la economía del cantón Paján y así aportar para su desarrollo productivo.

9. ¿Considera usted que con la enseña virtual se reducirá el nivel de analfabetismo en el Cantón Paján?

Tabla 9. Enseña virtual se reducirá el nivel de analfabetismo.

\begin{tabular}{|c|c|c|}
\hline Alternativa & Frecuencia & $\%$ \\
\hline Sí & 350 & 99 \\
\hline No & 2 & 1 \\
\hline Total & 352 & 100 \\
\hline
\end{tabular}

Fuente: Encuesta a productores y empleados del Gad Paján.

Elaborado por: Investigadores.

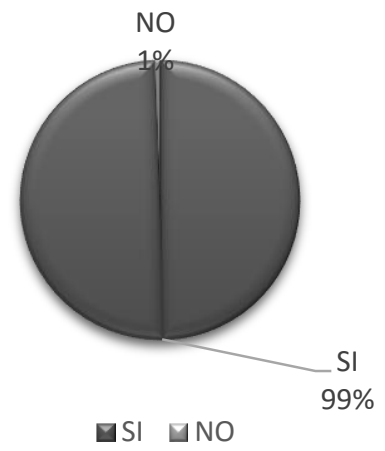

Gráfico 9. Enseña virtual se reducirá el nivel de analfabetismo.

Los resultados indican que el $99 \%$ de los productores y empleados del Gad Paján opinan que con la enseña virtual se reducirá el nivel de analfabetismos, mientras $1 \%$ no está de acuerdo.

Analizando los resultados obtenidos se pude concluir que los usuarios indican que la enseñanza virtual reducirá el nivel de analfabetismo, erradicando de esta forma uno de los problemas más comunes dentro del sector productivo den entorno, dando paso a obtener una población mejor informada, capacitada y emprendedora.

10. ¿Considera usted que con la herramientas tecnológica tendría un aprovechamiento sostenible y sustentable para el sector productivo?

Tabla 10. Aprovechamiento sostenible y sustentable para el sector productivo.

\begin{tabular}{|c|c|c|}
\hline Alternativa & Frecuencia & $\%$ \\
\hline Sí & 245 & 70 \\
\hline No & 107 & 30 \\
\hline Total & 352 & 100 \\
\hline
\end{tabular}

Fuente: Encuesta a productores y empleados del Gad Paján.

Elaborado por: Investigadores. 


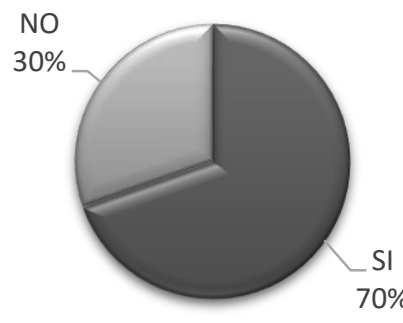

$\square \mathrm{SI} \quad \square \mathrm{NO}$

Gráfico 10. Aprovechamiento sostenible y sustentable para el sector productivo.

Los resultados indican que el $70 \%$ tendría aprovechamiento sostenible y sustentable para el sector productivo mientras $30 \%$ no está de acuerdo.

En conclusión, se podría acotar que la sustentabilidad y la sostenibilidad de las herramientas tecnológicas sería bien aprovechadas por el sector productivo para la mejora continua de sus productos debido a que estas herramientas facilitan la construcción de los conocimientos en base a estrategias.

\section{ESQUEMA GRÁFICO DE LA PROPUESTA "DESARROLLO DE LA PLATAFORMA VIRTUAL PARA EL DESARROLLO LOCAL"}

\subsection{ANTECEDENTES}

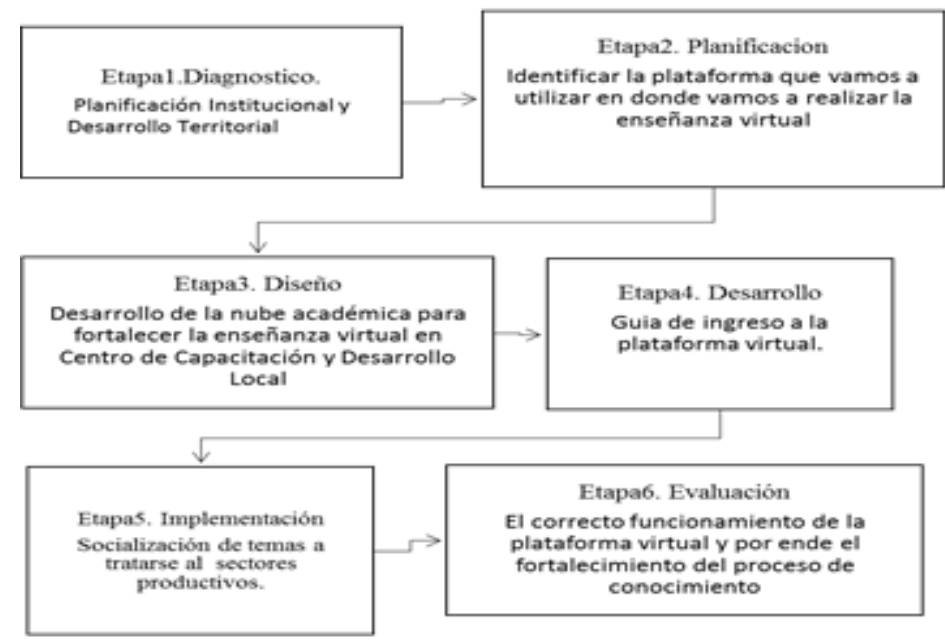

Figura 1. Esquema gráfico del desarrollo de la plataforma virtual en Claroline.

Fuente: elaboración propia.

El vertiginoso avance de los sistemas de información y comunicaciones en el Ecuador ha facilitado la automatización de los procesos, brindando confiabilidad, velocidad y precisión en el manejo de cada proceso automatizado. Desde este enfoque se puede decir que el uso eficiente y oportuno de la tecnología en procesos de capacitación para articular la gestión del conocimiento al sector productivo, 
define una gran ventaja para un desarrollo integral y participativo por parte de los productores identificados para el desarrollo de este proyecto.

El cantón Paján se encuentra ubicado al sur de la Provincia de Manabí, Pertenece a la Zona de Planificación 4 (Manabí-Sto. Domingo), y conforma el Distrito 13D09. Cuenta con una población total de 37.073 habitantes, de la cual el $47 \%$ son mujeres y el $53 \%$ son hombres, distribuidos el $81 \%$ en el sector rural y $19 \%$ en el área urbana, con una extensión territorial de 1.086 km2.

En las siguientes figuras se muestran las características socioeconómicas, nivel porcentual de analfabetismo y el proceso migratorio reciente en el cantón Paján según el INEC 2010, denotando valores importantes que se ha tomado en cuenta en la investigación.

\begin{tabular}{|c|c|c|c|c|c|}
\hline $\begin{array}{c}\text { TERRITORIOI } \\
\text { VARIABLE }\end{array}$ & Básica & Primaria & Secundaria & Bachillerato & Superior \\
\hline Lascano & 91,23 & 91,94 & 39,55 & 24,49 & 3,81 \\
\hline Guale & 89,83 & 93,51 & 46,95 & 29,66 & 2,27 \\
\hline Campozano & 87,27 & 93,33 & 43,38 & 30,30 & 3,86 \\
\hline Cascol & 87,17 & 89,22 & 44,39 & 27,86 & 3,95 \\
\hline Paján & 91,31 & 93,40 & 61,68 & 47,04 & 12,04 \\
\hline PAJAN & 89,41 & 92,28 & 47,19 & 31,87 & 5,19 \\
\hline MANABI & 91,68 & 92,83 & 63,74 & 50,51 & 20,16 \\
\hline
\end{tabular}

Figura 2. Características socioeconómicas por nivel de Educación. Fuente: INEC.

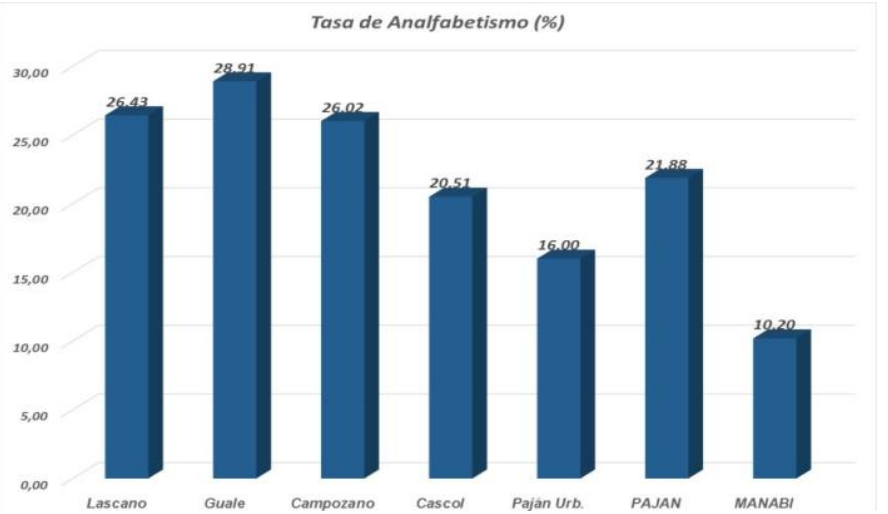

Figura 3. Tasa porcentual de analfabetismo en el Cantón Paján. Fuente: INEC.

\begin{tabular}{|c|c|c|}
\hline \multicolumn{2}{|c|}{ Movimientos migratorios } \\
\multicolumn{2}{|c|}{ Entradas y Salidas } \\
\hline \multirow{2}{*}{ Población residente actual } & Urbano & 6.731 \\
\cline { 2 - 3 } & Rural & 26.567 \\
\hline \multicolumn{2}{|c|}{ Población residente anterior } & 35.007 \\
\hline \multicolumn{2}{|c|}{ Población no migrante } & 31.797 \\
\hline \multirow{2}{*}{ Población inmigrante } & Urbano & 668 \\
\cline { 2 - 3 } & Rural & 833 \\
\hline \multicolumn{2}{|c|}{ Población emigrante } & 3.210 \\
\hline \multicolumn{2}{|c|}{ Migración neta } & $(1.709)$ \\
\hline \multicolumn{2}{|c|}{ Migración bruta } & 4.711 \\
\hline \multicolumn{2}{|c|}{} \\
\hline
\end{tabular}

Figura 4. Resumen del proceso migratorio reciente en el Cantón Paján. 
Fuente: INEC.Censo de población y vivienda 2010.

Dentro de las principales características territoriales que forman parte de las dificultades más notorias para el desarrollo del cantón se presenta la siguiente figura:

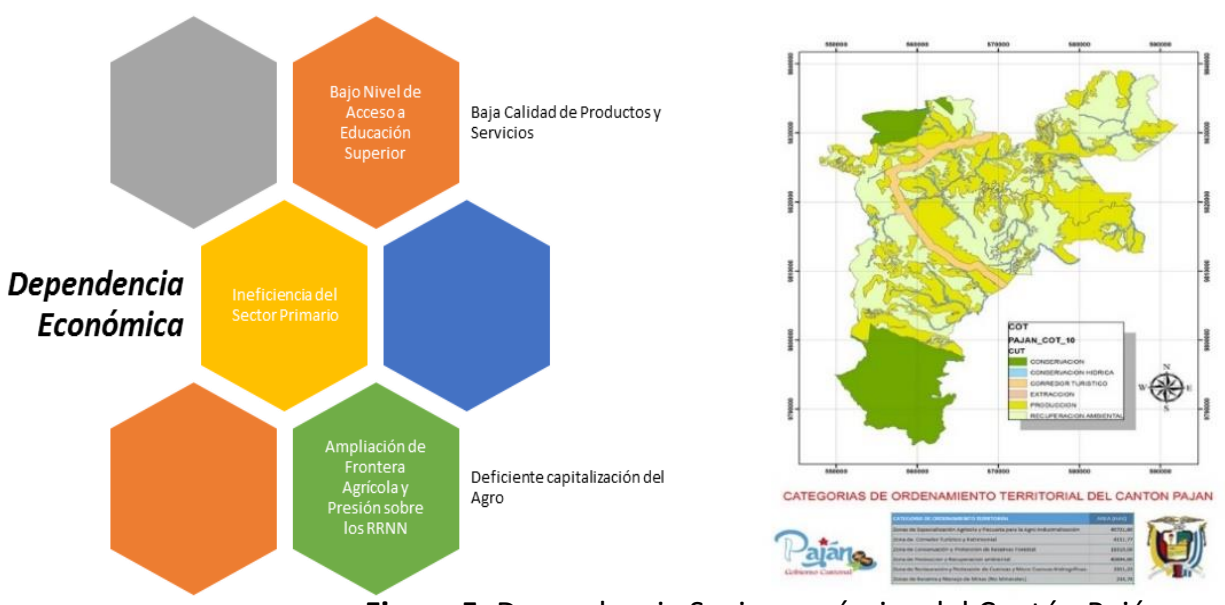

Figura 5. Dependencia Socioeconómica del Cantón Paján.

Se debe de tener en cuenta el papel preponderante que tienen que ejercer los Gobiernos Autónomos Descentralizados para lograr un verdadero desarrollo local en las diferentes áreas, que con la aplicación de nuevas tecnologías se conviertan en el eje matriz para el desarrollo de la matriz productiva y este sirva para alcanzar el Buen Vivir de acuerdo a la Constitución de la República del Ecuador.

La herramienta principal para el desarrollo de proyectos de investigación de las Universidades Ecuatorianas es el Plan de desarrollo y ordenamiento territorial (PDyOT) en donde mancomunadamente con el GAD serán participes y entes de transformación local, provincial y nacional. Desde ese punto de vista como visión para el año 2019, el cantón Paján será un territorio donde su desarrollo se basa en el manejo integral sustentable y sostenible de los recursos naturales, turismo responsable y con una economía solidaria dinámica, integrada, con actividades económicas diversificadas y cadenas productivas especializadas y eficientes, que produce y comercializa asociativamente productos con agregación de valor, rescata y fortalece sus raíces culturales y patrimoniales. También, mejora el acceso a servicios básico y educación superior y profesional, con servicios dignos y efectivos de salud, con un sistema vial, de tránsito y transporte que proporciona una movilidad y conectividad interna y externa que potencia el desarrollo económico del cantón, y servicio de energía eléctrica de buenas condiciones. Organiza y regula el uso y ocupación de suelo, mejora la gestión ingresos y el acceso a servicios municipales de la ciudadanía, y fortalece la participación ciudadana bajo un modelo de co-gestión y co-responsabilidad hacia el buen vivir de sus habitantes.

El rol de las Universidades para el desarrollo local conjuntamente con los GAD tiene que realizar la gestión del conocimiento e Innovación tecnológica acorde a la realidad local para el desarrollo académico - científico - económico y social auspiciado por la Universidad Estatal del Sur de Manabí, Carrera Ingeniería en Computación y Redes, Comisión de Investigación de la Carrera y docentes investigadores como actores principales para el avance del Sur de Manabí en el área de Innovación tecnológica. 
3C Tecnología (Edición 20) Vol.5 - № 4

Diciembre'16 - marzo'17, $36-55$

\section{(3c) tecnología}

Área de Innovación y Desarrollo, S.L.

ISSN: $2254-4143$

DOI: http://dx.doi.org/10.17993/3ctecno.2016.v5n4e20.36-55

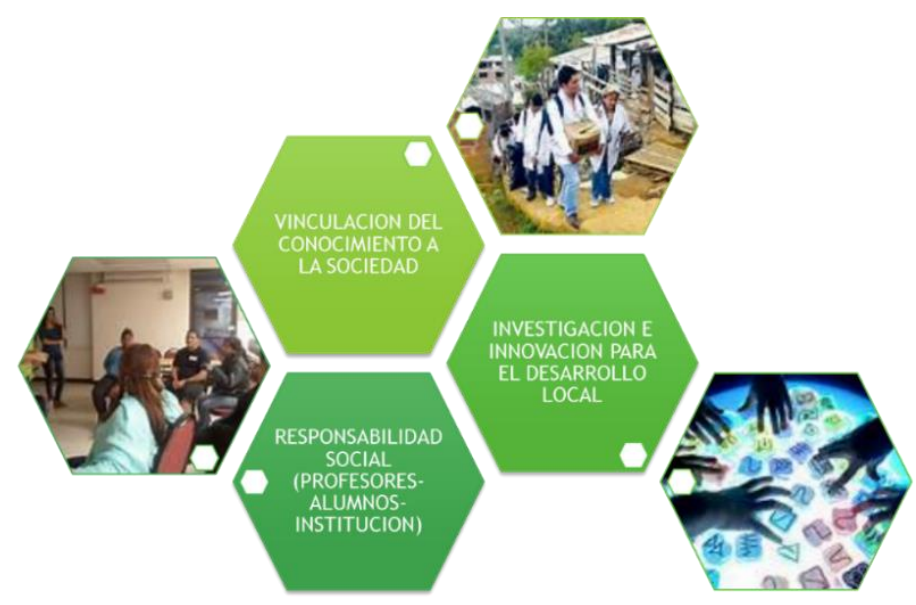

Figura 6. Rol de las Universidades para el desarrollo local sostenible y sustentable.

Es importante tener en cuenta las diferentes categorías de ordenamiento territorial para de esta manera fortalecer los proyectos de desarrollo mediante el uso de la tecnología.

\begin{tabular}{|l|r|}
\hline CATEGORIA DE ORDENAMIIENTO TERRITORIAL & AREA (HAS) \\
\hline Zonas de Especializaclón Agricola y Pecuaria para la Agro Industrialización & 40721,80 \\
\hline Zona de Corredor Turistico y Patrimonial & 4151,77 \\
\hline Zona de Conservación y Protección de Reservas Forestal & 18310,00 \\
\hline Zona de Produccion y Recuperacion ambiental & 40894,80 \\
\hline Zona de Restauración y Protección de Cuencas y Micro Cuencas Hidrogrificas. & 3351,23 \\
\hline Zonas de Reserva y Manejo de Minas (No Minerales) & 234,74 \\
\hline
\end{tabular}

Figura 7. Categorías de ordenamiento territorial del Cantón Paján.

Desde la perspectiva integradora - con un fuerte impacto social se desprende desde la UNESUM los Centros de Capacitación para el Desarrollo Local (CECADEL) y desde el GAD - PAJAN el Centro de Integración y Gestión del Conocimiento para el Desarrollo Local (CIGCDEL) como herramienta para el Desarrollo Local en el cantón Paján.
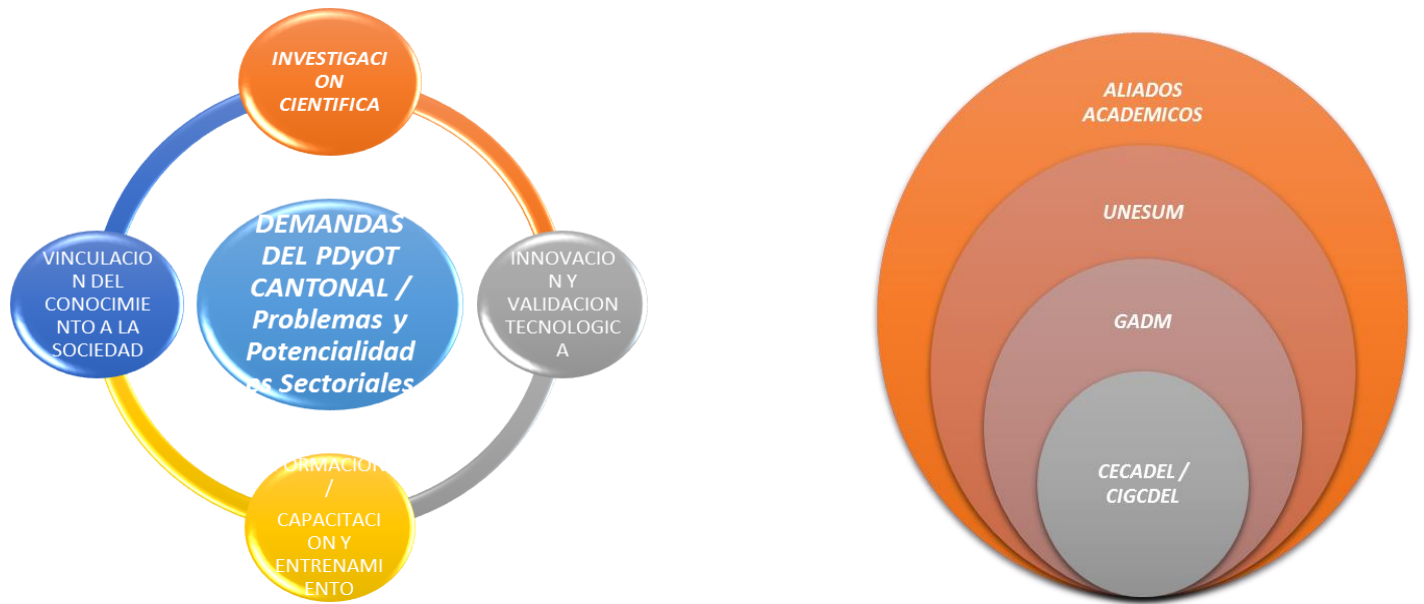

Figura 8. CECADEL/CIGCDEL - Demandas de Formación, Investigación e Innovación Tecnológica para el Desarrollo Local en el cantón Paján. 


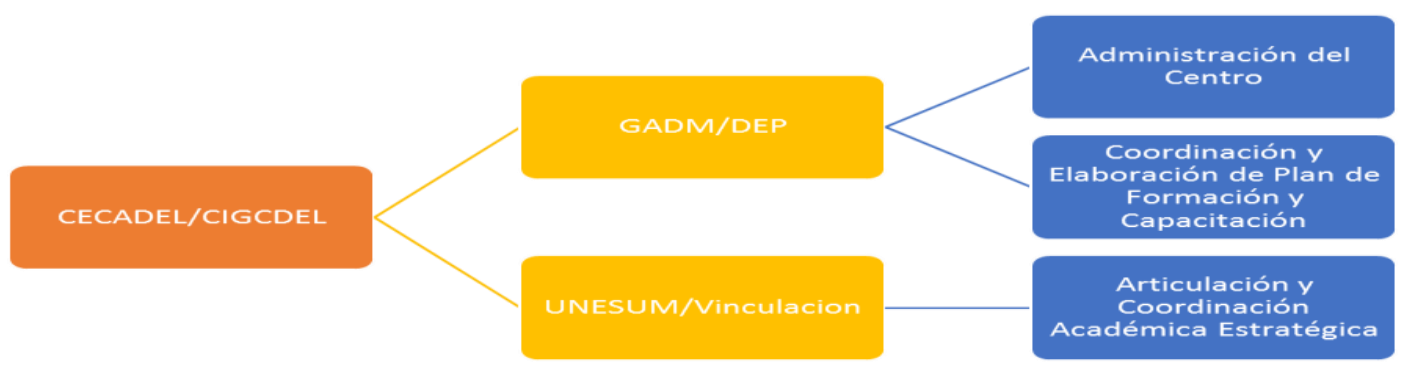

Figura 9. CECADEL / CIGCDEL estructura orgánica y operativa.

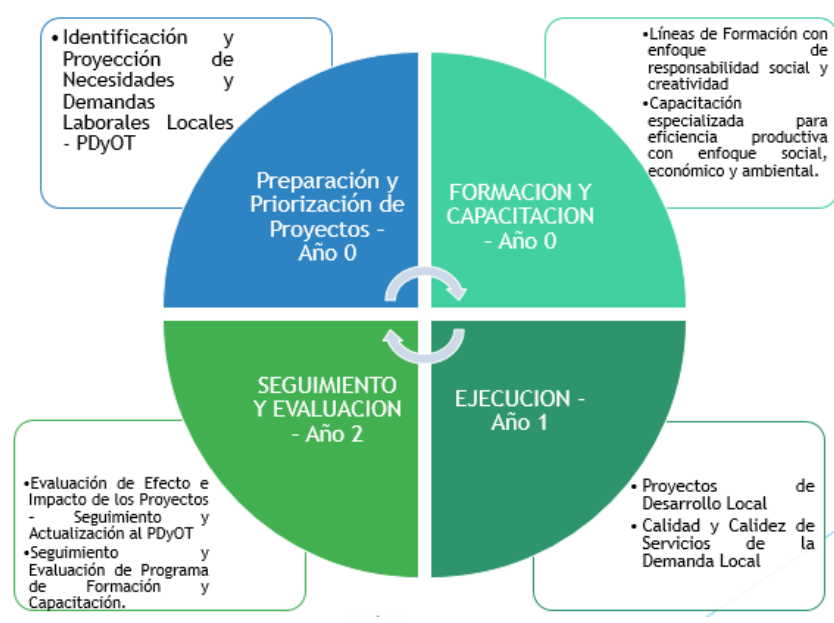

Figura 10. CECADEL / CIGCDEL Esquema de formación y capacitación.

De este diagnóstico de la realidad en cuestión se potencia el PDyOT para el desarrollo local sostenible y sustentable del Cantón Paján por medio de la utilización de las herramientas TI. En esta primera instancia el sistema se lo enfoco fortalecer capacitaciones / accesorias, acompañamiento técnico virtual para el desarrollo productivo agronómico sustentable.

\section{DESARROLLO E IMPLEMENTACIÓN DE LA PLATAFORMA VIRTUAL}

Para el desarrollo se trabajó con software libre plataforma de desarrollo Claroline, ya que cumple con las expectativas y fortalecerá la enseñanza virtual en el Centro de Capacitación y Desarrollo Local Paján de la Universidad Estatal del Sur de Manabí. 

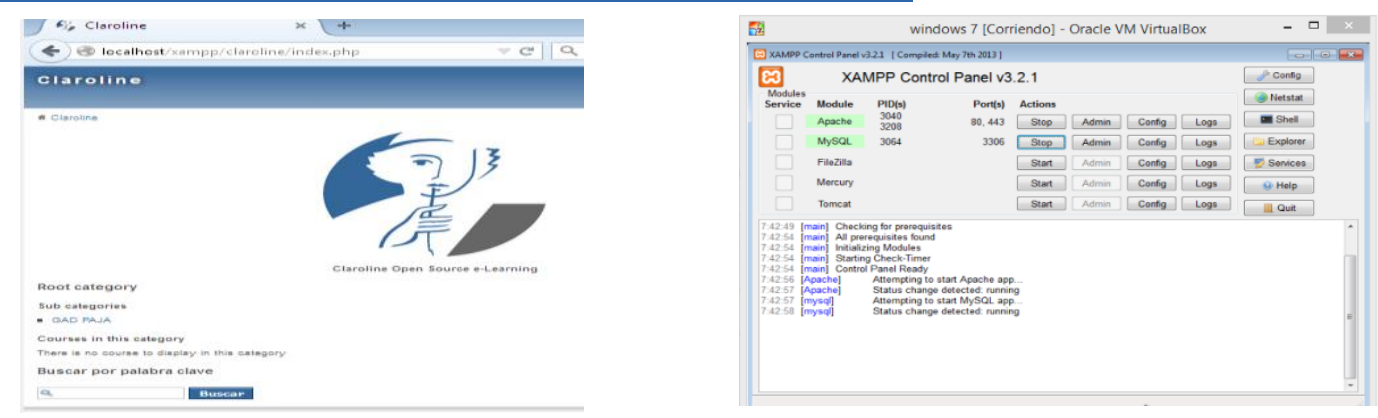

Figura 11. Plataforma y Servidor local (XAMPP).

Se utilizó un servidor local (XAMPP) y se desarrollaron diferentes tareas de acuerdo al objetivo de la plataforma:

Redactar el contenido de un curso, elaborar agendas con fechas, publicación de documento en distintos formatos (texto, doc., PDF, xIs, HTML, video), definir los objeto de Aprendizaje, elaborar ejercicios interactivos con preguntas opción múltiple, crear grupos de estudio, hacer que el aprendiz pueda enviar sus trabajos en lugares asignados, verificar que los estudiantes sigan un adecuado proceso en el desarrollo del aprendizaje.

De tal manera que se estructuraron jerárquicamente las diferentes actividades para la consecución de los resultados esperados:

Actividad 1. Se definieron los roles de los usuarios para el ingreso a la plataforma de la siguiente manera: Administrador: Se lo definió par que administra la plataforma, y controle todos los privilegios. Docente: Se lo definió para que reserve los derechos del estudiante, asignar los nuevos curso a crearse y modificar en todos sus contenido. Alumno: Se lo definió para que solo tenga acceso a los materiales y herramientas de los cursos creados por el docente.

Actividad 2. Se realizó la respectiva configuración de los diferentes roles a través de las diferentes herramientas de gestión.

Actividad 3. Se realizó la configuración del formulario para el desarrollo del curso de la siguiente manera:

- Título del curso: Agricultura Orgánica

- Código del curso: AOUNESUM - 2015

- Profesor: Ing. Carlos Fuentes Mendoza Mg. (Docente ESPAM)

- E-mail: carlosfuentes_m@hotmail.com

- Categoría: Administrador

- Acceso al curso: On - line

- Inscripción. Abierta 


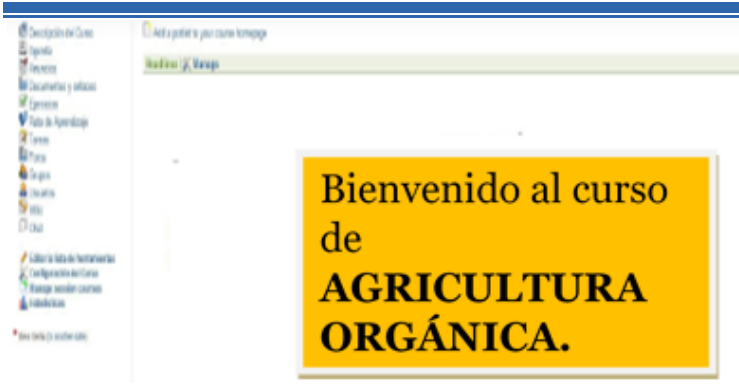

$+7 x$

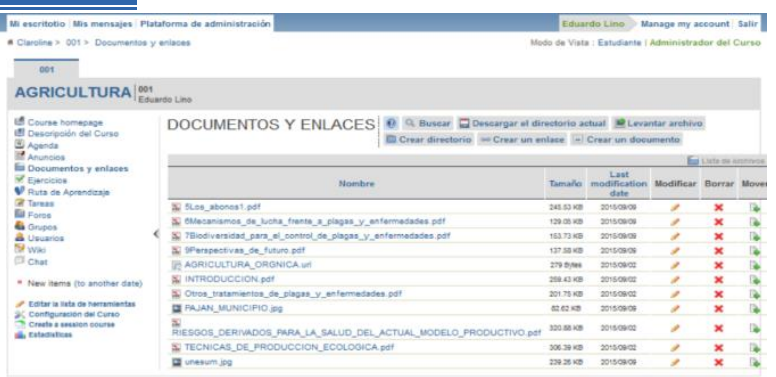

Figura 12. Pantalla principal del curso "Agricultura Orgánica" - Admin_Archivos_contenidos_curso.

Actividad 4. Se definieron los objetos de aprendizaje con el único fin de ordenar el proceso enseñanza / aprendizaje del curso brindando un orden pedagógico a cada contenido del curso. En donde se definieron: ejercicios previos, lectura recomendada, tareas asignadas y examen.

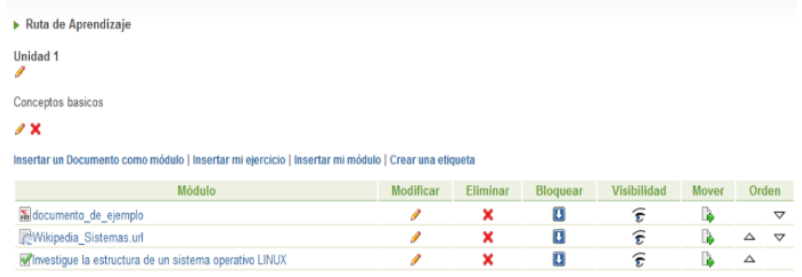

Figura 13. Objetos de Aprendizaje.

\section{Objetos de aprendizaje}

La preparación de los objetos de aprendizaje y las distintas actividades andragógicas depende la calidad académica y pedagógica para lograr buenos resultados. Por lo cual se debe desarrollar, diversas competencias, debido que se requiere estructurar un perfil que contempla las siguientes características:

1. Capacidad para la identificación de problemas de investigación y sus posibles soluciones en el marco de desarrollo local.

2. Habilidades para el desarrollo de contenidos teóricos - prácticos virtuales y presenciales de acuerdo al área de especialidad teniendo en consideración el enfoque multidisciplinario.

3. Conocimiento de metodología y técnicas de enseñanza para la creación de estrategias de aprendizaje orientadas a la reflexión, la síntesis y la capacidad cooperativa y colaborativa.

4. Seguimiento y evaluación de las prácticas innovadoras tecnológicas pertinentes para poder desarrollar objetos de aprendizaje para el desarrollo local.

5. El fortalecimiento de la interacción entre el usuario del objeto de aprendizaje y el objeto mismo por medio de la plataforma virtual.

Actividad 5. Se realizó el seguimiento de los objetos de aprendizajes para el desarrollo de competencias. 
Actividad 6. Se desarrolló del documento PDF. Es uno de los primeros paso de la ruta de aprendizaje de la Unidad 1.
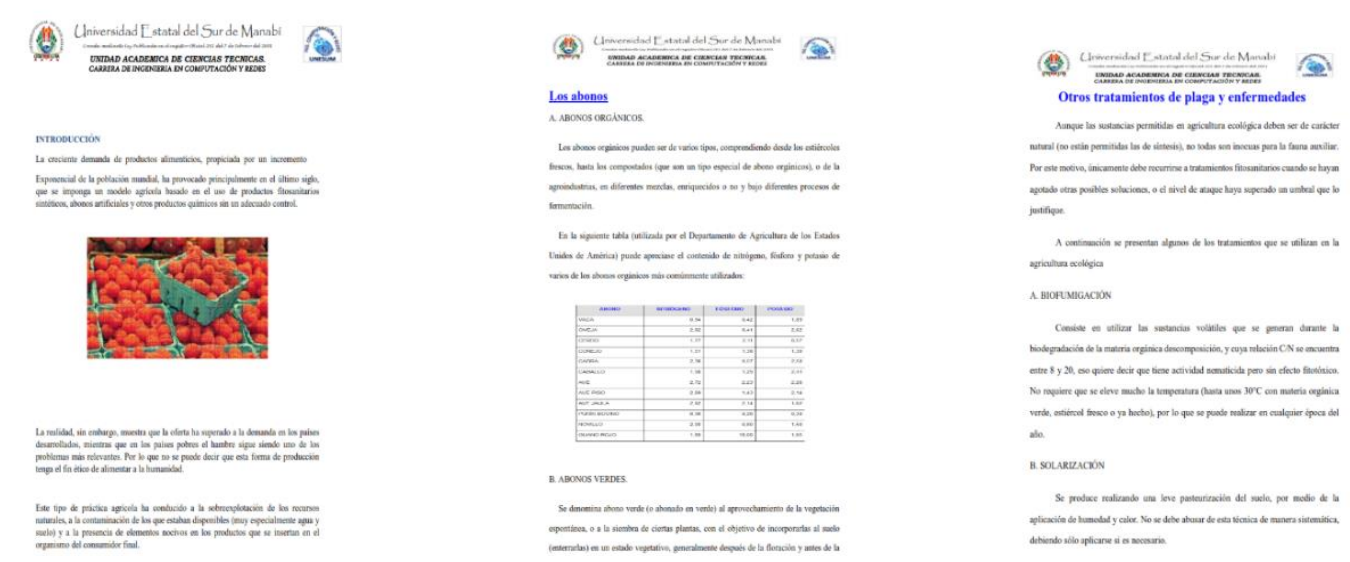

Figura 15. Curso Virtual de Agricultura Orgánica.

Actividad 7. Se motivó a la comunidad productora de Paján a participar en la socialización sobre el uso de la plataforma virtual y así fortalecer los conocimientos en el área productiva.

Actividad 8. Evaluación. En esta etapa luego de la capacitación a los usuarios en el manejo del aula virtual, se realizó la evaluación que estuvo a cargo de los involucrados (CECADEL, habitantes del sector productivo de Paján e investigador), además se identificó una diferencia positiva de manera gradual del sistema anterior al actual.

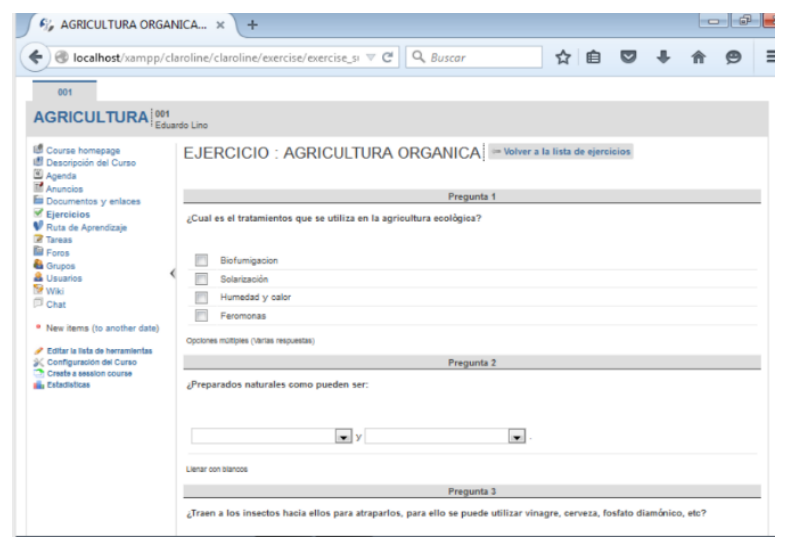

Figura 16. Proceso de Evaluación de la Plataforma.

\section{CONCLUSIONES}

Para el fortalecimiento de conocimientos técnicos / científicos dentro de los Gobiernos Autónomos Descentralizados y la generación de nuevos emprendimientos, proyectos vinculados para el desarrollo social con fuerte impacto en la comunidad, la plataforma virtual permitió mejorar el proceso de enseñanza / aprendizaje, fortalecer las horas extra clases de manera virtual, potenciando en primera instancia el área productiva local. 


\section{3c) tecnología}

3C Tecnología (Edición 20) Vol.5 - № 4

Diciembre'16 - marzo'17, $36-55$

Área de Innovación y Desarrollo, S.L.

ISSN: $2254-4143$

DOI: http://dx.doi.org/10.17993/3ctecno.2016.v5n4e20.36-55

La aplicación de la Plataforma Claroline cumple de manera directa con todos los criterios para el desarrollo de cursos virtuales y se acopla a la realidad local para mejorar resultados en el área de educación. Hasta ahora, el curso de Agricultura Orgánica ha cumplido con todas las expectativas y ha logrado capacitar a 352 agricultores esperando como último efecto la mejora de sus cosechas a través del uso de técnicas agrícolas en los campos.

El uso de la tecnología cada vez está creciendo a pasos agigantados, se estima que para el año 2019 un $50 \%$ de las clases serán de manera online, desde este punto de vista el CECADEL (Centro de capacitación y desarrollo local de la UNESUM), la Carrera Ingeniería en Computación y Redes y docentes Investigadores tienen grandes expectativas para el desarrollo de proyectos con fuerte impacto social en América del Sur específicamente en Ecuador, donde la Educación Superior está pasando por un proceso de trasformación académica - científica de manera acelerada.

El vínculo permanente entre Universidad - Estado - Empresa y Sociedad conllevan como resultado un verdadero desarrollo local. Por lo tanto, las Universidades son el ente motor para la trasformación de las ciudades. Actualmente, la aplicación de las TI conlleva a tener ciudades 100\% inteligentes en donde la tecnología esté vinculada en toda acción para mejorar los procesos sistemáticos del diario vivir.

\section{REFERENCIAS BIBLIOGRÁFICAS}

Castells, M. (2010). The Rise of the Network Society: With a New Preface. Volume I, Second edition With a new preface. 2a. edición.

Caicedo, C. (2016). Aplicativo móvil como estrategia de marketing para el impulso de la matriz productiva en el área turística. 3c Tecnología, 52.

Edel, R., y Navarro. (2010). Revista mexicana de investigación educativa. Recuperado de: $<$ http://www.scielo.org.mx/scielo.php?pid=S140566662010000100002\&script=sci arttext\&t|

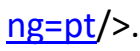

IAEN. (2011). Políticas públicas para la economía Social y Solidaria: líneas de investigación. SeminarioTaller Internacional Políticas públicas para la economía Social y Solidaria: líneas de investigación. Pp. 1-20. Quito.

Mell, P., y Grance, T. (2010). Draft NIST Working Definition of Cloud Computing.

Morales, J. (2003). Cloud computing: riesgos corporativos e implicaciones jurídicas. Recuperado de: $<$ http://www.garrigues.com/es/publicaciones/articulos/Paginas/Cloud-computing-riesgoscorporativos-e-implicaciones-juridicas.aspx/>.

Publishing, H. R. (2013). Universal Journal of Educational Research. Recuperado de: <http://www.hrpub.org/journals/article info.php?aid=1227/>.

Rekhy, R. (2011). mydigitalfc.com. Recuperado de: <www.mydigitalfc.com/op-ed/cloud-computingeducation-112/>. 
Requena, H. (2008). El modelo constructivista con las nuevas tecnologías: aplicado en el. Revista de Universidad y Sociedad del Conocimiento (RUSC).

Sánchez, S. (2015). Revista Iberoamericana para la Investigación y el Desarrollo Educativo. Recuperado de: <http://www.ride.org.mx/>.

Sierra, C. A. (2011). La educación virtual como favorecedora del aprendizaje autónomo.

Torres, L., Prieto, E., y López, L. (2012). Entornos Virtuales de Enseñanza. EDUTEC. Revista Electrónica de Tecnología Educativa. 